\title{
Analyzing the Mechanical Performance of Solid Oxide Fuel Cells at Interfacial Anode/Electrolyte Regions using Sub-Micron Resolution 3D X-Ray Computed Tomography
}

\author{
T. M. M. Heenan, J. B. Robinson, X. Lu, J. J. Bailey, D. J. L. Brett, P. R. Shearing \\ The Electrochemical Innovation Lab, Department of Chemical Engineering \\ University College London (UCL), London, WC1E 7JE, UK
}

\begin{abstract}
An inability to withstand rapid thermal cycling remains a shortcoming of the solid oxide fuel cell, resulting in start-up and shut-down times being prohibitively long. This work utilizes submicron resolution 3D X-ray CT reconstructions of a particularly problematic region due to a difference in thermal expansion properties within SOFCs, the anode-electrolyte interface. By examining the effects of thermal shock in $3 \mathrm{D}$, this work improves the understanding of the causes and effects of mechanical dynamics which are attributed to losses in electrochemical performance.
\end{abstract}

\section{Introduction}

Solid oxide fuel cells (SOFCs) potentially offer very high net efficiencies as well as fuel versatility, and so, are appealing for many technological applications. However, due to their high-temperature operation, differences in thermal properties such as the thermal expansion coefficient (TEC), particularly at the interface between neighboring layers such as the anode-electrolyte boundary, result in many thermally driven, mechanical degradation mechanisms. This inability to withstand rapid thermal shock limits thermal ramping, resulting in start-up and shut-down times being prohibitively long [1].

X-ray computed tomography (CT) has been employed extensively in the structural characterization of electrochemical devices and recent advancements have led to characterization being possible with the use of lab-based equipment, comparable to specialist facilities such as synchrotrons [2].

It is widely accepted that the density of percolated reaction sites, namely the triple phase boundary (TPB), strongly influences the electrochemical performance of the SOFC $[3,4]$. These sites are defined as a location where three transport networks meet: the gaseous, ionic and electronic; therefore, inspecting the effects of thermally induced structural degradation on the electrochemical activity networks can result in improved mitigation strategies for delaying/preventing degradation, ultimately extending cell lifetimes.

\section{Experimental}

A cylindrical sample, diameter ca. $800 \mu \mathrm{m}$, was extracted from an anode-supported NiO-8YSZ solid oxide half-cell comprising a thick anode (ca. $500 \mu \mathrm{m}$ ) with a thin (ca. 10 
$\mu \mathrm{m})$ 8YSZ electrolyte layer (Fuel Cell Materials, OH, USA). The sample was then mounted to an alumina tube using high-temperature cement (Aremco Products, New York, USA) before being subsequently milled to a smaller diameter suitable for highresolution imaging (diameter ca. $350 \mu \mathrm{m}$ ).

Once mounted and milled to a target diameter the sample was reduced to $\mathrm{Ni}$ $\mathrm{YSZ} / \mathrm{YSZ}$ in a tubular furnace under forming gas $\left(4 \% \mathrm{H}_{2}\right.$ in $\left.\mathrm{N}_{2}\right)$ for 2 hours at $800^{\circ} \mathrm{C}$ at a ramp-rate of $3^{\circ} \mathrm{C} \mathrm{min}^{-1}$. After reduction, the pristine microstructure was characterized using a VERSA 520 micro-CT X-ray instrument (Zeiss 520 VERSA, Carl Zeiss., CA, USA). The imaging conditions for all tomograms were kept constant throughout and are summarized in Table I.

TABLE I. X-ray Imaging Conditions

\begin{tabular}{lll}
\hline Parameter & Specifications & \\
\hline Radiograph Projections & 2401 & images \\
Exposure Time & 30 & seconds \\
Pixel Size & 0.395 & $\mu \mathrm{m}$ \\
Binning & 2 & - \\
Source Voltage & 90 & $\mathrm{kV}$ \\
Source Current & 90 & $\mu \mathrm{A}$ \\
Field of View & $384 \times 384$ & $\mu \mathrm{m} \times \mu \mathrm{m}$ \\
Sample Volume analyzed & $2 \times 10^{7}$ & $\mu \mathrm{m}^{3}$ \\
\hline
\end{tabular}

After the pristine microstructure had been captured by X-ray imaging the sample was reinserted into the tubular furnace and thermally cycled under forming gas to $750^{\circ} \mathrm{C}$ at a ramp-rate of $3^{\circ} \mathrm{C} \mathrm{min}^{-1}$ before being allowed to cool via natural convection. Once at room temperature the sample was imaged again under the same conditions as shown in Table I. This was then repeated for ramp-rates of 10,20 and $30^{\circ} \mathrm{C} \mathrm{min}{ }^{-1}$ acquiring tomograms sequentially at room temperature after each thermal cycle. The thermal-cycling conditions for all ramp-rates and resulting start-up times can be found in Table II.

TABLE II. Thermal Cycling Conditions

\begin{tabular}{ccc}
\hline Cycle Number $/$ no-units & Ramp Rate $/{ }^{\circ} \mathbf{C ~} \mathbf{~ m i n}^{-1}$ & Resulting Start-up time / min \\
\hline 1 & 3 & 242 \\
2 & 10 & 73 \\
3 & 20 & 36 \\
4 & 30 & 24 \\
\hline
\end{tabular}

The radiographic projections for each tomography were reconstructed using the inbuilt, cone-beam reconstruction software (Zeiss Reconstructor Scout-and-Scan software, Carl Zeiss, CA, USA). Due to the robustness of the sample mounting technique and the non-destructive nature of X-ray CT the same region of interest (ROI) was investigated for the duration of the study, allowing direct comparison of the microstructural evolutions with increased thermal shock. Segmentation was conducted using Avizo Fire software (Avizo, FEI, France). 

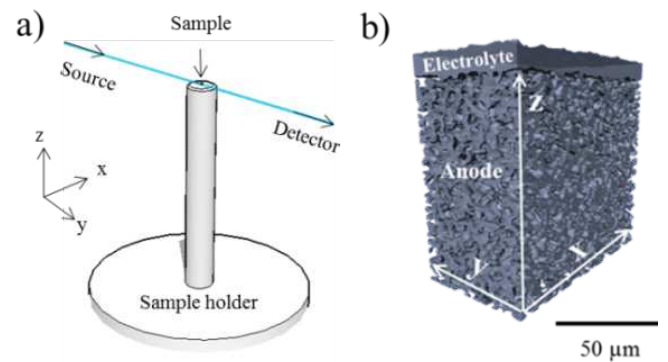

Figure 1. Experimental set-up for tomogram collection of a Ni-8YSZ anode supported solid oxide half-cell with 8YSZ electrolyte layer: a) X-ray path with respect to sample mount and holder, and b) sub-volume of the segmented interfacial region outlining the dense electrolyte layer atop the porous electrode.

\section{Results and Discussion}

By analyzing the same ROI after each thermal cycle with comparison to the fresh sample, the authors were able to inspect the thermally induced structural dynamics at both the anode-electrolyte interface and within the anode bulk. Displayed in Figure 2 are five $3 \mathrm{D}$ surface renderings generated from sub-volumes removed from the same ROI in each of the five tomograms.

Inspecting the anode-electrolyte interface by comparison of the fresh and final samples it is clear that the thermal expansion mismatch between the neighboring anode and electrolyte layers has resulted in a concaving of the electrolyte [Figure 2(f) and (g)]. Additionally, due to the large volumes which can be analyzed using this method the anode bulk can also be examined; Figure 3 displays crack propagation which has emerged within the final sample $\left(30^{\circ} \mathrm{C} \mathrm{min}^{-1}\right)$ likely due to the tensile stresses exerted within the anode during the compressive concaving of the electrolyte.

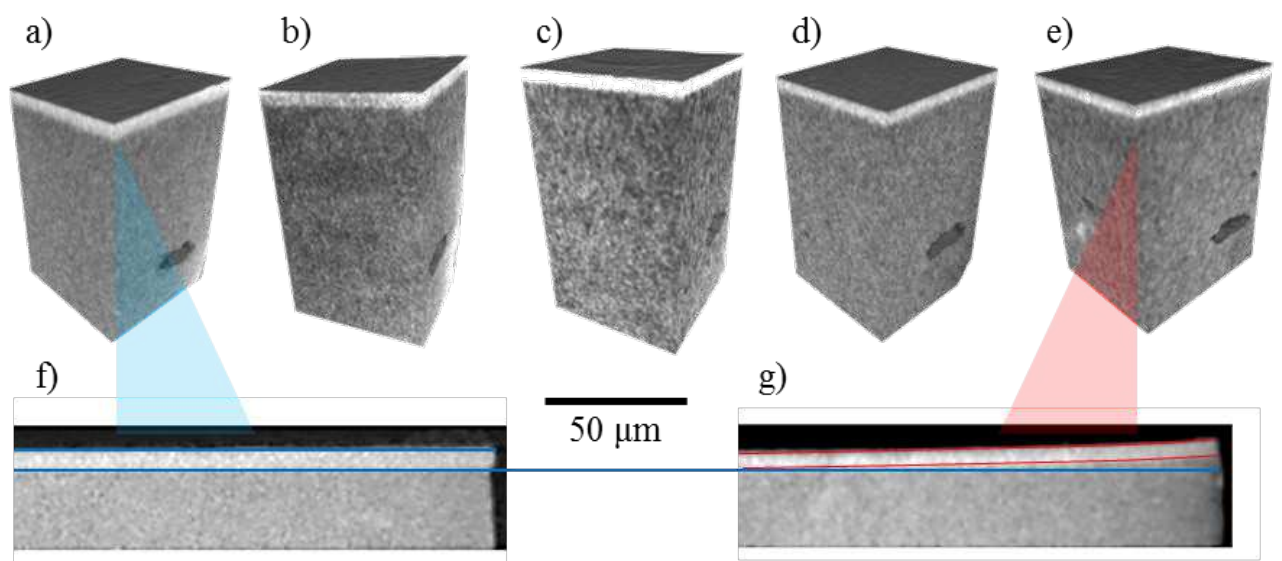

Figure 2. Multi-tomography analysis of a Ni-8YSZ/8YSZ solid oxide half-cell, exploring the effects of thermal ramp rate at interfacial regions: a) fresh sample, b) post $3{ }^{\circ} \mathrm{C} \mathrm{min}^{-1}$, c) post $10^{\circ} \mathrm{C} \mathrm{min}{ }^{-1}$, d) post $20^{\circ} \mathrm{C} \mathrm{min}^{-1}$ and e) post $30^{\circ} \mathrm{C} \mathrm{min}{ }^{-1}$, accompanying tomogram slice from the f) fresh and $\mathrm{g}$ ) post $30^{\circ} \mathrm{C} \mathrm{min}^{-1}$ interface. 


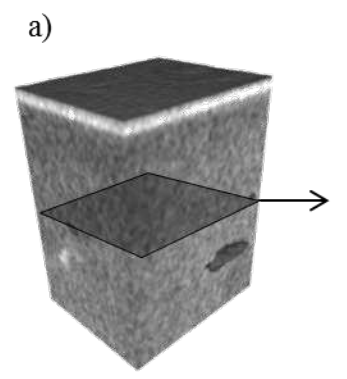

b)

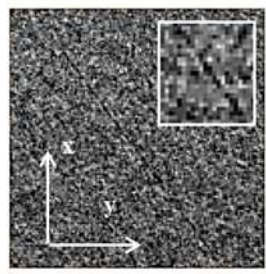

c)
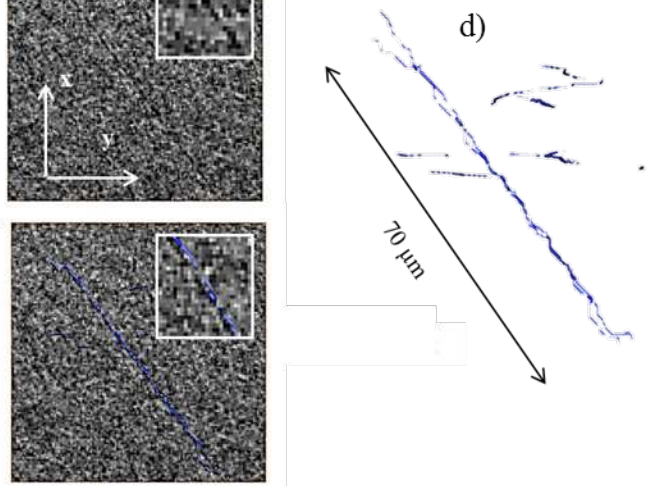

Figure 3. Initial signs of support layer degradation in the post $30^{\circ} \mathrm{C} \mathrm{min}{ }^{-1}$ sample: a) inspecting the anode support layer via b) a single greyscale tomogram slice, with c) crack propagation displayed in blue and d) accompanying crack segmentation.

These two mechanisms leading to mechanical degradation are likely to also affect the electrochemical performance of the cell; the TPB density is widely accepted to be strongly linked to the electrochemical performance. Figure 4 displays the quantification of the TPB locations for one slice of the interfacial sample and accompanying TPB density $\left(\rho_{T P B}\right)$ decline with increasing thermal cycles.
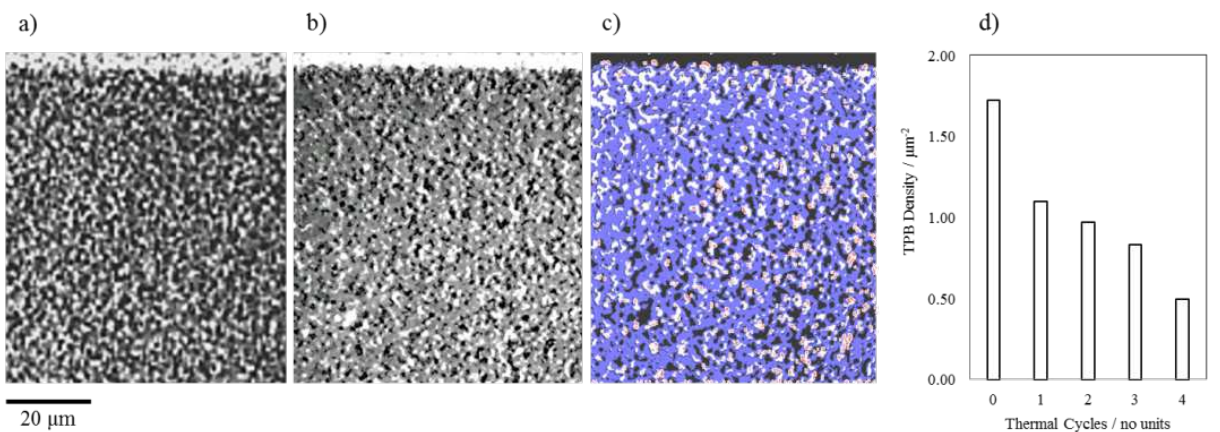

Figure 4. Three-phase segmentation of one tomogram slice for TPB quantification: a) raw tomogram slice, b) a non-local means filtered slice, c) segmented slice where black, white, blue and red display the YSZ, pore, Ni and TPB, d) the TPB density quantified for all four cycles in the same ROI.

\section{Conclusion}

This work provides operationally relevant insight into the effects of thermally driven degradation within SOFCs using sub-micron resolution, lab-based X-ray computed tomography. Initial findings suggest that exposure to thermal shock causes the anodeelectrolyte interface to undergo a thermally induced concaving, triggered by the mismatch in their thermal expansion coefficients. The effects of this concaving are seen, not only at the interfacial region but within the bulk of the anode support layer as well, in the form of tensile induced crack propagation which is a likely cause for reduction in percolated TPB density. 


\section{Acknowledgments}

The authors would like to acknowledge the EPSRC (EP/M014045/1), the Centre for Doctoral training (EP/L015749/1) and the Royal Academy of Engineering for financial support, access to the VERSA instrument was supported by the EPSRC (EP/N032888/1).

\section{References}

1. Atkinson, Alan, et al. "Advanced anodes for high-temperature fuel cells." Nature Materials, 3(1), 17-27 (2004).

2. Heenan, T. M. M., et al. "Three-Phase Segmentation of Solid Oxide Fuel Cell Anode Materials Using Lab Based X-ray Nano-Computed Tomography." Fuel Cells, 17(1), 75-82 (2017).

3. Shearing, P. R., et al. "3D reconstruction of SOFC anodes using a focused ion beam lift-out technique." Chemical Engineering Science, 64(17), 3928-3933 (2009).

4. Wilson, James R., et al. "Three-dimensional reconstruction of a solid-oxide fuelcell anode." Nature Materials, 5(7), 541-544 (2006). 